\title{
Characterization of Pv92, a Novel Merozoite Surface Protein of Plasmodium vivax
}

\author{
Seong-Kyun Lee ${ }^{1, \dagger}$, Bo Wang ${ }^{1,2, \dagger}$, Jin-Hee Han', Myat Htut Nyunt', Fauzi Muh', Patchanee Chootong ${ }^{3}$, \\ Kwon-Soo Ha ${ }^{4}$, Won Sun Park ${ }^{5}$, Seok-Ho Hong ${ }^{6}$, Jeong-Hyun Park ${ }^{7}$, Eun-Taek Han ${ }^{1, *}$

\begin{abstract}
'Department of Medical Environmental Biology and Tropical Medicine, School of Medicine, Kangwon National University, Chuncheon 24341, Korea; ${ }^{2}$ Department of Clinical Laboratory, The First Affiliated Hospital of Anhui Medical University, Hefei, Anhui, People's Republic of China; ${ }^{3}$ Department of Clinical Microbiology and Applied Technology, Mahidol University, Bangkok, Thailand; ${ }^{4}$ Department of Molecular and Cellular Biochemistry, ${ }^{5}$ Department of Physiology, ${ }^{6}$ Department of Internal Medicine, and ${ }^{7}$ Department of Anatomy, School of Medicine, Kangwon National University,
\end{abstract} Chuncheon 24341, Korea

\begin{abstract}
The discovery and understanding of antigenic proteins are essential for development of a vaccine against malaria. In Plasmodium falciparum, Pf92 have been characterized as a merozoite surface protein, and this protein is expressed at the late schizont stage, but no study of Pv92, the orthologue of Pf92 in P. vivax, has been reported. Thus, the protein structure of Pv92 was analyzed, and the gene sequence was aligned with that of other Plasmodium spp. using bioinformatics tools. The recombinant Pv92 protein was expressed and purified using bacterial expression system and used for immunization of mice to gain the polyclonal antibody and for evaluation of antigenicity by protein array. Also, the antibody against Pv92 was used for subcellular analysis by immunofluorescence assay. The Pv92 protein has a signal peptide and a sexual stage s48/45 domain, and the cysteine residues at the N-terminal of Pv92 were completely conserved. The N-terminal of Pv92 was successfully expressed as soluble form using a bacterial expression system. The antibody raised against Pv92 recognized the parasites and completely merged with PvMSP1-19, indicating that Pv92 was localized on the merozoite surface. Evaluation of the human humoral immune response to Pv92 indicated moderate antigenicity, with $65 \%$ sensitivity and $95 \%$ specificity by protein array. Taken together, the merozoite surface localization and antigenicity of Pv92 implicate that it might be involved in attachment and invasion of a merozoite to a new host cell or immune evasion during invasion process.
\end{abstract}

Key words: Plasmodium vivax, Pv92, merozoite surface protein, antigenicity

\section{INTRODUCTION}

Plasmodium vivax caused an estimated 13.8 million malaria cases in 2015, and was responsible for about a half of all malaria cases outside Africa. In addition, the total number of malaria deaths due to $P$. vivax was estimated to be between 1,400 and 14,900 [1]. Understanding and characterizing the functions of Plasmodium spp. proteins would facilitate development of a vaccine against malaria. Although potential vaccine candidates have been identified using bioinformatics tools and the PlasmoDB database, and the number of candidates has been increasing, only 3 P. vivax candidate antigens for a malaria vaccine are at present in preliminary (phase I) clinical

- Received 1 May 2016, revised 29 May 2016, accepted 29 May 2016.

*Corresponding author (ethan@kangwon.ac.kr)

${ }^{\dagger}$ These authors contributed equally to this work.

(c) 2016, Korean Society for Parasitology and Tropical Medicine

This is an Open Access article distributed under the terms of the Creative Commons Attribution Non-Commercial License (http://creativecommons.org/licenses/by-nc/4.0) which permits unrestricted non-commercial use, distribution, and reproduction in any

medium, provided the original work is properly cited. trials [2-4]. The proteins involved in invasion such as attachment, junction formation, and internalization of host erythrocytes have been identified as important vaccine candidates located on the surface or in the apical organelles of merozoites [5-7].

To date, 12 members of the $s 48 / 45$ protein family (Pfs230, Pfs48/45, Pfs230p, Pfs47, P52, P36, Pf41, Pf38, Pf12, P12p, Pf92, and sequestrin) have been identified in $P$. falciparum parasites. These antigens are likely present on the surface of merozoites (the asexual stage parasite that invades erythrocyte) and gametocytes (the sexual stage parasite in mosquitoes) [8,9]. One of the s48/45 protein family containing s48/45 domains, Pfs230, is expressed on the gametocyte surface, and specific antibodies against the $\$ 48 / 45$ domain containing 6-cysteine (Cys) residues blocks oocyst production. In addition, the absence of Pfs 230 showed significant inhibition of oocyst production as well as mosquito infectivity [10-13]. Therefore, the Pfs48/45 antigens such as Pfs 230 are potential vaccine candidates for blocking transmission of malaria $[14,15]$. 
In a previous report, Pf92 of the s48/45 protein family, which contains a single s48/45 domain, was expressed during merozoite stages, and contained a glycosylphosphatidylinositol-anchor (GPI-anchor) domain at the C-terminal. In addition, the central peptides of Pf 22 showed high binding ability to erythrocytes, and a rabbit polyclonal anti-Pf92 antiserum strongly inhibited invasion of the P. falciparum parasite into erythrocytes. In addition, Pf92 has been confirmed as a merozoite surface protein at the schizont stage $[16,17]$. However, homologues of Pf92 in other Plasmodium spp. remain unclear. Previous studies identified and characterized the localization and antigenicity of several 6-Cys proteins in $P$. vivax, such as Pv41 and Pv12, which are expressed on the surface and rhoptry, respectively, of merozoites during the asexual cycle $[18,19]$. Antibodies against Pvs48/45 and Pvs47 expressed in gametocytes of $P$. vivax reduced oocyst development, consistent with $P$. falciparum [20].

To date, few features of Pv92 are known; for instance, it belongs to the 6-Cys protein family and its expression level is low [21]. However, its localization and antigenicity have not been documented. Therefore, the Pv92 was expressed using an Escherichia coli protein expression system. The recombinant Pv92 protein was subjected to evaluation of antigenicity using a protein array and immunized into mice to obtain antiserum, which was used to confirm the subcellular localization of Pv92 in the late schizont.

\section{MATERIALS AND METHODS}

\section{P. vivax sample collection and serum preparation}

A total of 32 blood samples were collected from patients with confirmed vivax malaria via microscopy at local health centers and clinics in Gangwon Province, an endemic area in the Republic of Korea. A total of 23 blood samples were collected from healthy donors who were confirmed vivax malaria negative via microscopy, and were used as controls. The Institutional Review Board at Kangwon National University Hospital approved this study.

\section{Bioinformatics analysis of Pv92}

Gene sequence data and gene expression profiles of Pv92 as well as homologue genes were obtained from the PlasmoDB website (http://plasmodb.org; accession no. PVX_115165). Protein domains were further predicted using the Simple Modular Architecture Research Tool (SMART) (http://smart. embl-heidelberg.de/) [22,23]. The gene sequence data of Pv92 and those of other Plasmodium spp. were aligned using the DNASATR software (Lasergene, Madison, Wisconsin, USA).

\section{Genomic DNA isolation}

One of the above Korean P. vivax isolates was subjected to genomic DNA extraction using QIAamp DNA Blood Mini Kits (Qiagen, Hilden, Germany) according to the manufacturer's instructions, and used for PCR amplification of the Pv92 gene. The purified genomic DNA was eluted in $200 \mu$ of elution buffer and stored at $-20^{\circ} \mathrm{C}$ until use.

\section{Cloning and protein expression of Pv92}

The N-terminus of pv92 gene was amplified by PCR from genomic DNA and cloned using the pEXP5-NT/TOPO ${ }^{\circledR} \mathrm{TA}$ Expression Kit (Thermo Fisher, Massachusetts, USA). For gene amplification, the following pair of primers was designed: Pv92-F (5'-GTGTCCCTTGCAGGGGAGTT-3') and Pv92-R (5'-TTAGCTAGGGGGCGCCCCTT-3'). The sequences underlined are terminator codons. The PCR reaction system contained 0.5 U Platinum Taq DNA Polymerase High Fidelity (Invitrogen, Carlsbad, California, USA), $0.2 \mu \mathrm{M}$ of sense and antisense primer, 50 ng of genomic DNA, $200 \mu \mathrm{M}$ deoxyribonucleotide triphosphates (dNTPs), and $\mathrm{MgSO}_{4}$ to a final concentration of $2.0 \mathrm{mM}$. PCR amplification from genomic DNA was carried out under the following cycling conditions for $38 \mathrm{cy}-$ cles: $94^{\circ} \mathrm{C}$ for $20 \mathrm{sec}, 60^{\circ} \mathrm{C}$ for $30 \mathrm{sec}, 72^{\circ} \mathrm{C}$ for $1 \mathrm{~min}$, and a final extension step at $72^{\circ} \mathrm{C}$ for $10 \mathrm{~min}$. The amplified gene was purified by PCR clean-up kit (Macherey-Nagel, Düren, Germany) according to the manufacturer's instructions. The purified gene was incubated with PEXP5-NT/TOPO vector according to the manufacturer's instructions, and then the cloned plasmid DNA was transformed into TOP10 competent cells by the heat-shock method. The DNA sequence was confirmed using the ABI PRISM 310 Genetic Analyzer and a Big Dye Terminator v1.1 Cycle sequencing kit (Applied Biosystems, Forster City, California, USA).

The clone was transformed into BL21(DE3) competent cells (Invitrogen) for protein expression. Production of the recombinant protein was induced by isopropyl- $\beta$ - D-thiogalactopyranoside (IPTG). The lysis of E. coli was carried out by lysis buffer $(1 \mathrm{mg} / \mathrm{ml}$ lysozyme, $1 \mathrm{mM}$ PMSF, 1mM DTT, $10 \mu \mathrm{M}$ Leupeptine) and sonication, and the lysate centrifuged to gain the soluble fraction. The soluble fraction was incubated with Ni-NTA (Qiagen) for overnight and the recombinant protein 
was eluted according to the manufacturer's instructions. The purified recombinant protein was confirmed by SDS-PAGE and Western blot analysis.

\section{Production of mouse immune sera}

To generate antibodies, $20 \mu \mathrm{g}$ of the purified protein Pv92 was used for immunization of 3 female BALB/c mice (5-weekold) (Daehan Biolink Co., Eumsung, Korea) with Freund's complete adjuvant, followed by $20 \mu \mathrm{g}$ with Freund's incomplete adjuvant. Immunization was performed 3 times at 2-week intervals. The animal experiment was approved by the Institutional Ethics Committee and we followed the Ethical Guidelines for Animal Experiments of Kangwon National University.

\section{SDS-PAGE and Western blot analysis}

The recombinant Pv92 protein was separated by $12 \%$ SDSPAGE under reducing conditions. The separated protein was transferred onto a $0.45 \mu \mathrm{m}$ PVDF membrane (Millipore, Billerica, Massachusetts, USA) in semi-dry transfer buffer ( $50 \mathrm{mM}$ Tris, $190 \mathrm{mM}$ glycine, $3.5 \mathrm{mM}$ SDS, 20\% methanol) at a constant $400 \mathrm{~mA}$ for $40 \mathrm{~min}$ using a semi-dry blotting system (ATTO Corp., Tokyo, Japan). After blocking with 5\% skim milk in PBS-T (0.5\% v/v Tween-20 in $1 \times$ PBS), anti-histidine antibody (Qiagen), mouse immune serum, or mixed patient sera diluted 1:100 in PBS-T and secondary IRDye ${ }^{\circledR}$ goat anti-mouse (1:5,000 dilution) or IRDye ${ }^{\circledast}$ goat anti-human $(1: 20,000)$ (Li$\mathrm{COR}^{\circledR}$ Bioscience, Lincoln, Nebraska, USA) antibodies were used to detect recombinant protein. The results were visualized using the Odyssey infrared imaging system (Li-COR ${ }^{\circledR}$ Bioscience) and analyzed using the Odyssey software (Li-COR ${ }^{\circledR}$ Bioscience).

\section{Indirect immunofluorescence assay (IFA)}

Blood smears were prepared for IFA as described previously [19]. Slides smeared with schizont enriched blood were fixed with ice-cold acetone for $3 \mathrm{~min}$, dried, and stored at $-80^{\circ} \mathrm{C}$ until use. The frozen slides were thawed on silica gel blue (Samchun Chemical, Pyeongtaek, Korea) and blocked with 5\% BSA in $\mathrm{PBS}$ at $37^{\circ} \mathrm{C}$ for $30 \mathrm{~min}$. The slides were incubated with 1:100 dilutions of primary antibodies (mouse anti-Pv92 and rabbit anti-PvMSP1-19) at $37^{\circ} \mathrm{C}$ for $1 \mathrm{hr}$. The slides were then stained with Alexa Fluor 488-conjugated goat anti-rabbit IgG or Alexa Fluor 546-conjugated goat anti-mouse IgG secondary antibodies (Invitrogen) and the nuclei were stained with 4',6-diamidino-2-phenylindole (DAPI; Invitrogen) at $37^{\circ} \mathrm{C}$ for $30 \mathrm{~min}$. The slides were mounted in ProLong Gold antifade reagent (Invitrogen) and visualized under oil immersion using a confocal laser scanning FV200 microscope (Olympus, Tokyo, Japan). Images were captured using the FV10-ASW 3.0 viewer software.

\section{Assessment of antigenicity using protein array}

The amine-coated slides were prepared as described previously [4]. A total of 32 or 20 serum samples from vivax patients or healthy persons, respectively, were used to evaluate the antigenicity of Pv92. One-hundred nanograms of Pv92 protein were spotted on each well of a slide, and incubated for $2 \mathrm{hr}$ at $37^{\circ} \mathrm{C}$. After incubation, the wells were blocked with 5\% BSA in PBS for $1 \mathrm{hr}$ at $37^{\circ} \mathrm{C}$. Then, slides were incubated with human individual sera at a 1:10 dilution in PBS for $1 \mathrm{hr}$ at $37^{\circ} \mathrm{C}$ and then with Alexa Fluor 546 goat anti-human $\operatorname{IgG}$ in PBS-T for $1 \mathrm{hr}$ at $37^{\circ} \mathrm{C}$ for visualization. The slides were scanned in a fluorescence scanner (Perkin Elmer, Boston, Massachusetts, USA) [24]. The mean fluorescence intensities (MFI) of spots were quantified by the fixed circle method using the ScanArray Express software version 4.0 (PerkinElmer). The positive cut-off MFI value was set as the MFI of serum samples from healthy individuals plus 2 SDs [25].

\section{Statistical analysis}

The data were analyzed with GraphPad Prism (GraphPad Software, San Diego, California, USA) and SigmaPlot (Systat Software Inc., San Jose, California, USA). Mann-Whitney U tests were used to determine the significance of differences between the means of the groups. Values of $P<0.05$ were considered to indicate statistical significance.

\section{RESULTS}

\section{Bioinformatics analysis of Pv92 sequence}

Pv92 gene sequence information encoded by PVX_115165 on chromosome 11 retrieved from PlasmoDB revealed that the ORF of the Pv92 encodes an 888-amino-acid protein with a predicted molecular mass of $98.51 \mathrm{kDa}$. The $\mathrm{Pv} 92$ protein is encoded by a single exon gene, and consists of a signal peptide (amino acid [aa] 1-23), Pfs48/45 domain (aa 571-963), and GPI-anchor (aa 861-888) (Fig. 1A). Orthologues of Pv92 were found in 5 Plasmodium spp. including P. falciparum IT and 3D7. To further characterize the Pv92 protein, the N-terminal 


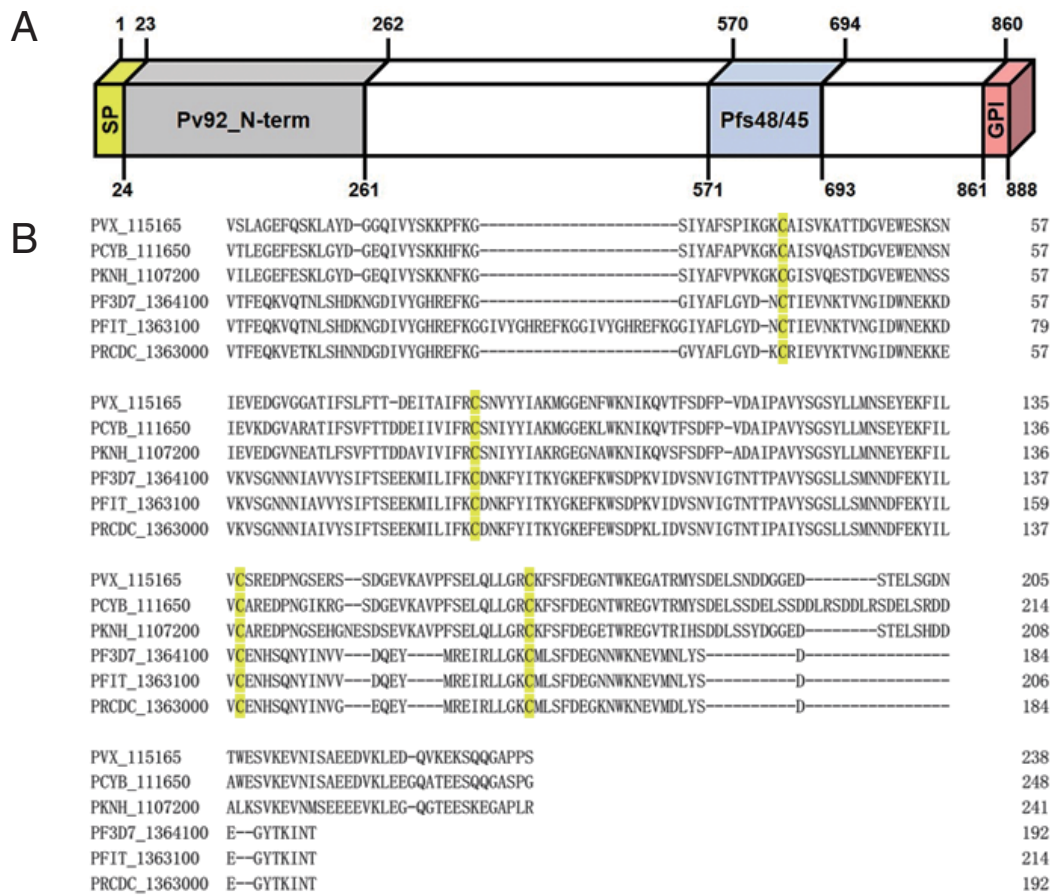

Fig. 1. Schematic diagram and amino acid sequence alignment of Pv92 with homologues in other Plasmodium spp. (A) Schematic diagram of Pv92. The Pv92 protein comprises 888 amino acids. Indicated are the signal peptide (aa 1-23), Pfs48/45 domain (aa 571-963), and GPI-anchor (aa 861-888). The N-terminal (aa 24-261) was constructed for protein expression. (B) Amino acid sequence alignment of N-terminal of Pv92 with 5 homologues in other Plasmodium spp. Cysteine residues are highlighted in yellow.
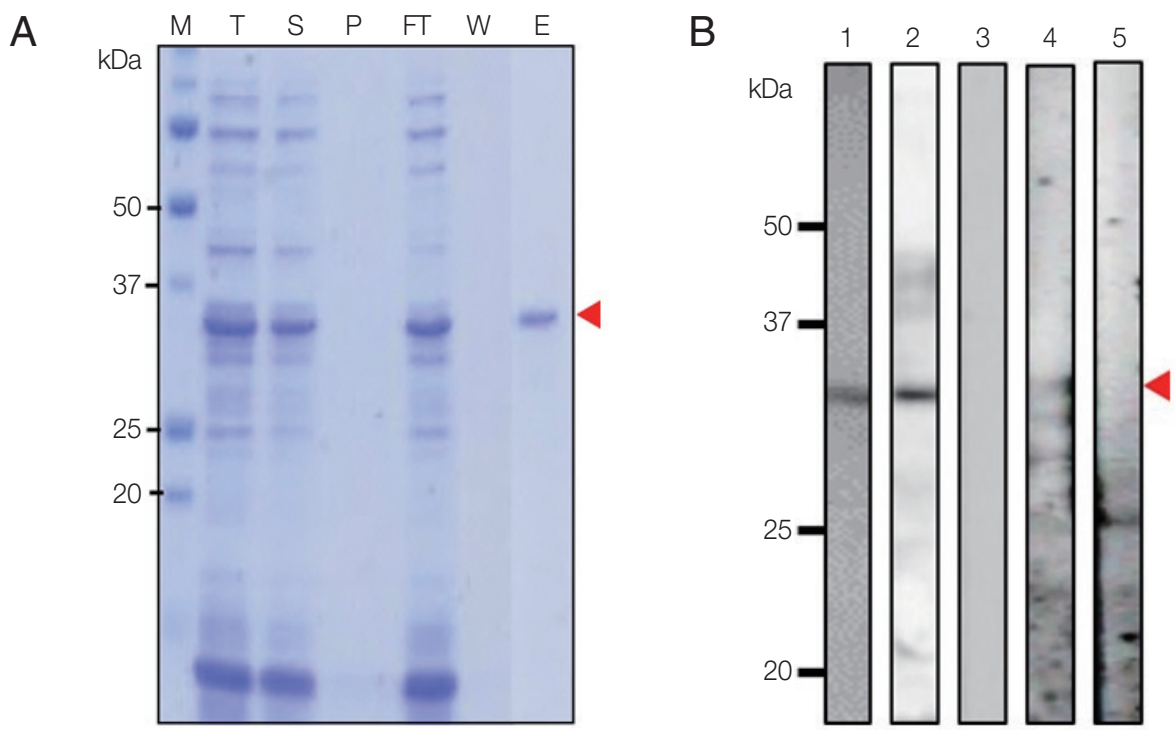

Fig. 2. Recombinant Pv92 protein expression, purification, and Western-blot analysis. (A) Purified N-terminal of Pv92 protein. M, protein marker; arrow heads, purified Pv92; T, total fraction; S, soluble fraction; P, pellet fraction; Ft, flow-through; W, washing fraction; E, elution fraction. (B) Western-blot analysis of Pv92 using an anti-His antibody (lane 1), mouse immune serum (lane 2), pre-immune mouse serum (lane 3), pooled vivax patient sera (lane 4), and malaria-naïve human serum samples (lane 5). Arrowheads indicate Pv92.

(aa 24-261) was aligned with orthologues in other Plasmodium

the species examined (Fig. 1B). spp. Five cysteine residues were completely conserved across 

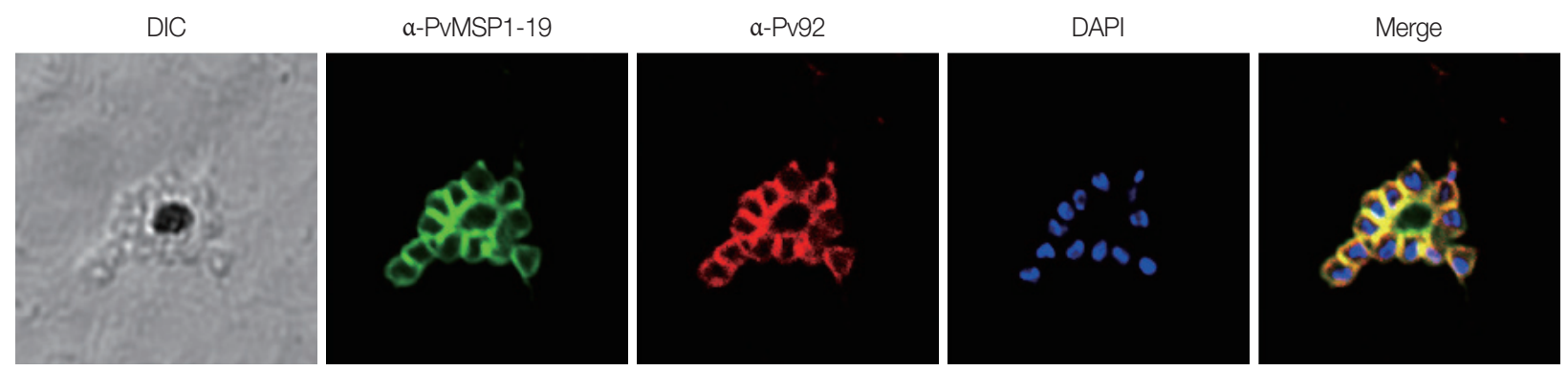

Fig. 3. Localization of Pv92 at the mature schizont stage. The parasites were co-labeled with antisera against Pv92 (red color), PvMSP1-19 (merozoite surface marker, green color), and DAPI (nuclei marker, blue color).

\section{Expression and purification of Pv92, and western blotting analysis of immune serum against Pv92}

The N-terminal of Pv92 was successfully expressed and purified under non-denaturing condition using an E. coli protein expression system, and the purity was confirmed by SDS-PAGE (Fig. 2A). Pv92 migrated as a single band of $~ 33 \mathrm{kDa}$ under reducing condition; the predicted size of Pv92 was slightly smaller, at $\sim 28 \mathrm{kDa}$. Polyclonal antibodies against the protein were produced by immunizing mice with purified Pv92, and the serum was used to assess recombinant protein expression and localization at $P$. vivax schizonts. The serum against Pv92 and $P$. vivax patient serum successfully recognized Pv92 in western blotting analysis (Fig. 2B).

\section{Pv92 locates on the merozoite surface of parasites}

To determine the localization of the Pv92 protein in mature schizonts, IFA was carried out using anti-Pv92 and -PvMSP1-19 sera. In parasites, Pv92 was localized to the surface of each merozoite (Fig. 3). As Pv92 was highly expressed only at the schizont stage throughout the $48 \mathrm{hr}$ intraerythrocytic cycle, its localization was compared with the merozoite surface protein PvMSP1-19. The signal of Pv92 completely merged with that of MSP1-19, demonstrating that Pv92 is a merozoite surface protein.

\section{Humoral immune response to Pv92}

To further evaluate the humoral immune response to Pv92, a protein array was used to screen the presence of antibodies in individual human sera. Antibody responses against Pv92 in serum samples from 32 patients infected with P. vivax and 20 healthy individuals were determined. The responses of patients exposed to $P$. vivax were significantly higher than those of naive subjects (Fig. 4, $P<0.0001$ ). The prevalence of antiPv92 antibodies was also determined, indicating a sensitivity

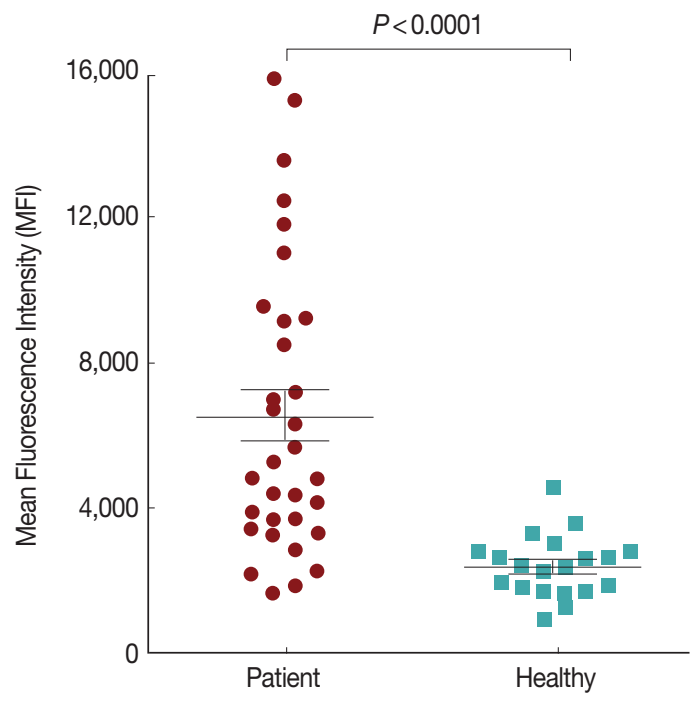

Fig. 4. Humoral responses to Pv92 as determined by protein arrays. Pv92 was reacted with the sera of vivax malaria patients (positive) or healthy individuals (negative) from Korea. The prevalence of anti-Pv92 lgG differed significantly between vivax patients and healthy individuals $(P<0.0001)$. $P$-value was calculated using Mann-Whitney $U$ tests. Bar indicates the mean plus SD.

of $65 \%$ (21 positives of 32 vivax samples) and specificity of $95 \%$ (19 negatives of 20 healthy samples) (Table 1).

\section{DISCUSSION}

A deeper understating of antigens involved in invasion is essential for development of malaria vaccines. To identify promising $P$. vivax vaccine candidates, antigenic proteins expressed during the asexual stage were investigated using an immunoproteomics profiling method [4]. Difficulty expressing Pfs 230 and Pfs48/45 has been an obstacle to understand these antigens $[9,14,26]$. Previous data on Pf92 showed that it localized to the merozoite surface and central parts of erythrocyte binding sites $[16,17]$. However, the orthologue of Pf92 in P. vivax, 
Table 1. IgG responses to Pv92 in serum samples from patients and healthy individuals

\begin{tabular}{lcccccc}
\hline \multirow{2}{*}{ Samples } & \multicolumn{2}{c}{ No. of samples } & & $\begin{array}{c}\text { Sensitivity or } \\
\text { specificity (\%) }\end{array}$ & 95\% Cl (\%) & MFI \\
\cline { 2 - 3 } & Positive & Negative & & 65 & $48.3-79.5$ & $6,594 \pm 4,028^{a}$ \\
\hline Vivax patients $(n=32)$ & 21 & 11 & 95 & $76.3-99.1$ & $2,410 \pm 851$ \\
Healthy individuals $(n=20)$ & 1 & 19 & & 95 & \\
\hline
\end{tabular}

$\mathrm{Cl}$, confidence interval; MFI, mean fluorescence intensity.

${ }^{a} P$-value $<0.0001$.

Pv92, remains uncharacterized. Thus, herein, the N-terminal of Pv92 was expressed and purified using an E. coli protein expression system, and then characterized. The expressed Pv92 protein reacted with the pooled vivax patient sera, indicating that it was immunogenic and may be important role in merozoite attachment and invasion of erythrocytes. To evaluate the antigenicity of Pv92 using individual vivax patient serum, a protein array was carried out using the sera of 32 patients and 20 healthy individuals. Among them, $65 \%$ of the serum of vivax patients (Table 1) showed seropositivity, which is lower than that of PvMSP1-19 [18]. In P. falciparum, Pf92 is localized on the merozoite surface at the late schizont stage and is also present at the ring stage [16]. In a previous report of proteins of the s48/45 family in P. vivax, the antigenicity of Pv41 was similar to that of the merozoite surface protein Pv92 [18], while another s48/45 family protein, Pv12, which is a rhoptry protein, showed lower antigenicity than Pv92 [19], indicating that Pv92 is a moderate antigenic protein among s48/45 family in P. vivax. To determine the localization of Pv92 in parasites, IFA was performed with rabbit anti-PvMSP1-19 as a positive control and it is localized on the merozoite surface on the schizont stage parasites, indicating that Pv92 may play an important role for attachment and invasion of erythrocyte in blood stage.

In a recent study, Pv12 and Pv42, the s48/45 family protein, were produced high $\operatorname{IgG}$ antibody responses in naturally acquired immune response analysis of filed isolates [27]. However, Pv92 was observed the lowest IgG antibody responses in naturally $P$. vivax-exposed individuals among 34 merozoite proteins of P. vivax [27]. We could not compared with our study and other merozite antigens because expression methods (bacterial or eukaryotic expression), serum samples (patients, age, etc.) and screening methods (ELISA or protein array) with diverse sensitivities were affected on candidate selection. Although the technical difficulties such as long-term culture system of $P$. vivax, it will be required to analyze function of Pv92 during erythrocyte invasion of blood stage parasites and determine the potential of these proteins as serological marker.

\section{ACKNOWLEDGEMENTS}

Authors are grateful to Jung-Yeon Lee for technical assistance. This work was supported by a National Research Foundation of Korea (NRF) grant funded by the Korea Government (MSIP) (NRF-2014R1A2A1A11052079), by the Basic Science Research Program through the National Research Foundation of Korea (NRF) funded by the Ministry of Science, ICT \& Future Planning (2015R1A4A1038666) and by 2014 Research Grant from Kangwon National University (no. C1010825-0101). The funders had no role in the study design, data collection and analysis, decision to publish, or preparation of the manuscript.

\section{CONFLICT OF INTEREST}

The authors declare that they have no competing interests.

\section{REFERENCES}

1. WHO. World Malaria Report (2015). 2015.

2. Mueller I, Shakri AR, Chitnis CE. Development of vaccines for Plasmodium vivax malaria. Vaccine 2015; 33: 7489-7495.

3. Herrera S, Corradin G, Arevalo-Herrera M. An update on the search for a Plasmodium vivax vaccine. Trends Parasitol 2007; 23: 122-128.

4. Chen JH, Jung JW, Wang Y, Ha KS, Lu F, Lim CS, Takeo S, Tsuboi T, Han ET. Immunoproteomics profiling of blood stage Plasmodium vivax infection by high-throughput screening assays. J Proteome Res 2010; 9: 6479-6489.

5. Arevalo-Pinzon G, Curtidor H, Vanegas M, Vizcaino C, Patarroyo MA, Patarroyo ME. Conserved high activity binding peptides from the Plasmodium falciparum Pf34 rhoptry protein inhibit merozoites in vitro invasion of red blood cells. Peptides 2010; 31: 1987-1994.

6. Cowman AF, Crabb BS. Invasion of red blood cells by malaria parasites. Cell 2006; 124: 755-766.

7. Jaskiewicz E, Graczyk J, Rydzak J. Proteins involved in invasion 
of human red blood cells by malaria parasites. Postepy Hig Med Dosw (Online) 2010; 64: 617-626.

8. Gerloff DL, Creasey A, Maslau S, Carter R. Structural models for the protein family characterized by gamete surface protein Pfs230 of Plasmodium falciparum. Proc Natl Acad Sci USA 2005; 102: 13598-13603.

9. Sanders PR, Cantin GT, Greenbaum DC, Gilson PR, Nebl T, Moritz RL, Yates JR, 3rd, Hodder AN, Crabb BS. Identification of protein complexes in detergent-resistant membranes of Plasmodium falciparum schizonts. Mol Biochem Parasitol 2007; 154: 148-157.

10. Eksi S, Czesny B, van Gemert GJ, Sauerwein RW, Eling W, Williamson KC. Malaria transmission-blocking antigen, Pfs230, mediates human red blood cell binding to exflagellating male parasites and oocyst production. Mol Microbiol 2006; 61: 991998.

11. Rener J, Graves PM, Carter R, Williams JL, Burkot TR. Target antigens of transmission-blocking immunity on gametes of Plasmodium falciparum. J Exp Med 1983; 158: 976-981.

12. Vermeulen AN, Roeffen WF, Henderik JB, Ponnudurai T, Beckers PJ, Meuwissen JH. Plasmodium falciparum transmission blocking monoclonal antibodies recognize monovalently expressed epitopes. Dev Biol Stand 1985; 62: 91-97.

13. Quakyi IA, Carter R, Rener J, Kumar N, Good MF, Miller LH. The 230-kDa gamete surface protein of Plasmodium falciparum is also a target for transmission-blocking antibodies. J Immunol 1987; 139: 4213-4217.

14. Chowdhury DR, Angov E, Kariuki T, Kumar N. A potent malaria transmission blocking vaccine based on codon harmonized full length Pfs48/45 expressed in Escherichia coli. PLoS One 2009; 4: e6352.

15. Williamson KC. Pfs230: from malaria transmission-blocking vaccine candidate toward function. Parasite Immunol 2003; 25: 351-359.

16. Obando-Martinez AZ, Curtidor H, Arevalo-Pinzon G, Vanegas M, Vizcaino C, Patarroyo MA, Patarroyo ME. Conserved high activity binding peptides are involved in adhesion of two detergentresistant membrane-associated merozoite proteins to red blood cells during invasion. J Med Chem 2010; 53: 3907-3918.

17. Sanders PR, Gilson PR, Cantin GT, Greenbaum DC, Nebl T, Carucci DJ, McConville MJ, Schofield L, Hodder AN, Yates JR 3rd, Crabb BS. Distinct protein classes including novel merozoite surface antigens in Raft-like membranes of Plasmodium falci- parum. J Biol Chem 2005; 280: 40169-40176.

18. Cheng Y, Lu F, Tsuboi T, Han ET. Characterization of a novel merozoite surface protein of Plasmodium vivax, Pv41. Acta Trop 2013; 126: 222-228.

19. Li J, Ito D, Chen JH, Lu F, Cheng Y, Wang B, Ha KS, Cao J, Torii M, Sattabongkot J, Tsuboi T, Han ET. Pv12, a 6-Cys antigen of Plasmodium vivax, is localized to the merozoite rhoptry. Parasitol Int 2012; 61: 443-449.

20. Tachibana M, Suwanabun N, Kaneko O, Iriko H, Otsuki H, Sattabongkot J, Kaneko A, Herrera S, Torii M, Tsuboi T. Plasmodium vivax gametocyte proteins, Pvs48/45 and Pvs47, induce transmission-reducing antibodies by DNA immunization. Vaccine 2015; 33: 1901-1908.

21. Hostetler JB, Sharma S, Bartholdson SJ, Wright GJ, Fairhurst RM, Rayner JC. A library of Plasmodium vivax recombinant merozoite proteins reveals new vaccine candidates and protein-protein interactions. PLoS Negl Trop Dis 2015; 9: e0004264.

22. Schultz J, Milpetz F, Bork P, Ponting CP. SMART, a simple modular architecture research tool: identification of signaling domains. Proc Natl Acad Sci USA 1998; 95: 5857-5864.

23. Letunic I, Doerks T, Bork P. SMART: recent updates, new developments and status in 2015. Nucleic Acids Res 2015; 43: D257D260.

24. Neeley ES, Kornblau SM, Coombes KR, Baggerly KA. Variable slope normalization of reverse phase protein arrays. Bioinformatics 2009; 25: 1384-1389.

25. Chen JH, Wang Y, Ha KS, Lu F, Suh IB, Lim CS, Park JH, Takeo S, Tsuboi T, Han ET. Measurement of naturally acquired humoral immune responses against the C-terminal region of the Plasmodium vivax MSP1 protein using protein arrays. Parasitol Res 2011; 109: 1259-1266.

26. Tachibana M, Wu Y, Iriko H, Muratova O, MacDonald NJ, Sattabongkot J, Takeo S, Otsuki H, Torii M, Tsuboi T. N-terminal prodomain of Pfs230 synthesized using a cell-free system is sufficient to induce complement-dependent malaria transmissionblocking activity. Clin Vaccine Immunol 2011; 18: 1343-1350.

27. França CT, Hostetler JB, Sharma S, White MT, Lin E, Kiniboro B, Waltmann A, Darcy AW, Li Wai Suen CS, Siba P, King CL, Rayner JC, Fairhurst RM, Mueller I. An antibody screen of a Plasmodium vivax antigen library identifies novel merozoite proteins associated with clinical protection. PLoS Negl Trop Dis 2016; 10: e0004639. 